PROCEEDINGS OF THE

AMERICAN MATHEMATICAL SOCIETY

Volume 134, Number 7, Pages 2131-2137

S 0002-9939(06)08230-X

Article electronically published on January 31, 2006

\title{
SPECTRUM OF BILATERAL SHIFTS WITH OPERATOR-VALUED WEIGHTS
}

\author{
A. BOURHIM
}

(Communicated by Joseph A. Ball)

Abstract. We describe the spectrum of bilateral operator-weighted shifts.

\section{INTRODUCTION}

Throughout this note, $\mathcal{L}(\mathcal{H})$ will denote the algebra of all bounded linear operators acting on a complex Hilbert space $\mathcal{H}$ and $l^{2}(\mathbb{Z}, \mathcal{H})$ will be the usual Hilbert space of all square summable sequences $\left(x_{n}\right)_{n \in \mathbb{Z}}$ whose elements are in $\mathcal{H}$. For an operator $T \in \mathcal{L}(\mathcal{H})$, we denote by $\sigma(T), \sigma_{s u}(T), \sigma_{a p}(T)$, and $\sigma_{p}(T)$ the spectrum, the surjectivity spectrum, the approximate point spectrum, and the point spectrum of $T$, respectively. We also denote by $r(T)$ the spectral radius of $T$, and by $q(T)$ the minimum modulus of $\sigma(T)$, that is,

$$
q(T)= \begin{cases}0 & \text { if } T \text { is not invertible } \\ \frac{1}{r\left(T^{-1}\right)} & \text { if } T \text { is invertible }\end{cases}
$$

Moreover, we let $m(T):=\inf \{\|T x\|:\|x\|=1\}$ stand for the lower bound of $T$ and recall that the sequence $\left(m\left(T^{n}\right)^{\frac{1}{n}}\right)_{n \geq 1}$ converges and its limit, denoted by $r_{1}(T)$, equals its supremum.

Let $\left(A_{n}\right)_{n \in \mathbb{Z}}$ be a two-sided sequence of uniformly bounded invertible operators in $\mathcal{L}(\mathcal{H})$, and let $\left(B_{n}\right)_{n \in \mathbb{Z}}$ be the sequence given by

$$
B_{n}:= \begin{cases}A_{n-1} A_{n-2} \ldots A_{1} A_{0} & \text { if } n>0, \\ 1 & \text { if } n=0, \\ A_{n}^{-1} A_{n+1}^{-1} \ldots A_{-2}^{-1} A_{-1}^{-1} & \text { if } n<0 .\end{cases}
$$

The corresponding bilateral operator-weighted shift on $l^{2}(\mathbb{Z}, \mathcal{H})$ is defined by

$$
S\left(\ldots, x_{-2}, x_{-1},\left[x_{0}\right], x_{1}, x_{2}, \ldots\right):=\left(\ldots, A_{-2} x_{-2},\left[A_{-1} x_{-1}\right], A_{0} x_{0}, A_{1} x_{1}, \ldots\right),
$$

where for an element $x=\left(\ldots, x_{-2}, x_{-1},\left[x_{0}\right], x_{1}, x_{2}, \ldots\right) \in l^{2}(\mathbb{Z}, \mathcal{H})$, we denote by $\left[x_{0}\right]$ the 0 -th coordinate of $x$.

In [5, Theorem 2], Herrero provided some spectral inclusions for hyponormal bilateral operator-weighted shifts. He then deduced that the spectrum of a hyponormal bilateral operator-weighted shift of multiplicity $k$ (i.e., $\mathcal{H}=\mathbb{C}^{k}$ ) is the

Received by the editors December 3, 2004 and, in revised form, February 23, 2005.

2000 Mathematics Subject Classification. Primary 47B37; Secondary 47A10, 47A11.

Key words and phrases. Bilateral weighted shifts, spectrum, local spectrum.

(C)2006 American Mathematical Society Reverts to public domain 28 years from publication 
union of at most $k$ annuli (see [5, Corollary 3]). In [2, Ben-Artzi and Gohberg introduced the concepts of Bohl exponent and canonical splitting projection to describe the spectrum and the essential spectrum of $S$ when $\mathcal{H}=\mathbb{C}^{k}$ and

$$
\max \left(\sup _{n \in \mathbb{Z}}\left\|A_{n}\right\|, \sup _{n \in \mathbb{Z}}\left\|A_{n}{ }^{-1}\right\|\right)<+\infty .
$$

But these two concepts are quite complicated and are not easy to apply in concrete situations except when the corresponding weight sequence is particularly special. In [6], Li, Ji and Sun first proved that each operator-weighted shift, $S$, of finite multiplicity $k$, is unitarily equivalent to a $k \times k$ upper-triangular operator matrix, $\mathcal{M}$, whose coefficients are scalar weighted shifts. Therefore, they completely describe the essential spectrum of $S$ in terms of the scalar weighted shifts situated on the diagonal of $\mathcal{M}$.

In 4, the author described the local spectrum of $S$ at most points of $l^{2}(\mathbb{Z}, \mathcal{H})$ and used some tools from local spectral theory to describe completely the spectrum of all bilateral operator-weighted shifts satisfying Dunford's condition $(C)$ (see 4 , Theorem 4.2]).

The main goal of the present note is to describe the spectrum of bilateral operator-weighted shifts that have no restriction on the corresponding weight sequences. We feel that our approach, which is based on local spectral theory, is simpler and more accessible than the approach of Ben-Artzi and Gohberg.

\section{MAIN RESUlts}

We begin this section by fixing some notation that will play a crucial role in the rest of this note. For an injective bilateral scalar weighted shift $T$ with a bounded weight sequence $\left(v_{n}\right)_{n \in \mathbb{Z}}$, we set

$$
\begin{array}{lll}
r^{-}(T) & =\lim _{n \rightarrow+\infty}\left\{\sup _{k>0} \prod_{i=k+1}^{n+k}\left|v_{-i}\right|\right\}^{\frac{1}{n}}, & r^{+}(T)=\lim _{n \rightarrow+\infty}\left\{\sup _{k \geq 0} \prod_{i=k}^{n+k-1}\left|v_{i}\right|\right\}^{\frac{1}{n}}, \\
r_{1}^{-}(T)=\lim _{n \rightarrow+\infty}\left\{\inf _{k>0} \prod_{i=k+1}^{n+k}\left|v_{-i}\right|\right\}^{\frac{1}{n}}, & r_{1}^{+}(T)=\lim _{n \rightarrow+\infty}\left\{\inf _{k \geq 0}^{n+k-1} \prod_{i=k}^{n}\left|v_{i}\right|\right\}^{\frac{1}{n}}, \\
r_{2}^{-}(S)=\liminf _{n \rightarrow+\infty}\left\{\prod_{i=1}^{n}\left|v_{-i}\right|\right\}^{\frac{1}{n}}, & r_{2}^{+}(S)=\liminf _{n \rightarrow+\infty}\left\{\prod_{i=0}^{n-1}\left|v_{i}\right|\right\}^{\frac{1}{n}}, \\
r_{3}^{-}(S)=\limsup _{n \rightarrow+\infty}\left\{\prod_{i=1}^{n}\left|v_{-i}\right|\right\}^{\frac{1}{n}}, & r_{3}^{+}(S)=\limsup _{n \rightarrow+\infty}\left\{\prod_{i=0}^{n-1}\left|v_{i}\right|\right\}^{\frac{1}{n}} .
\end{array}
$$

Keep in mind that

$$
r_{1}(T)=\min \left(r_{1}^{-}(T), r_{1}^{+}(T)\right)
$$

$$
r(T)=\max \left(r^{-}(T), r^{+}(T)\right),
$$

and

$$
r_{1}^{ \pm}(T) \leq r_{2}^{ \pm}(T) \leq r_{3}^{ \pm}(T) \leq r^{ \pm}(T) .
$$

The spectrum of $T$ and its parts are described in terms of the above quantities.

Theorem 2.1. The following assertions hold.

(i) The spectrum of $T$ is given by

$$
\sigma(T)=\{\lambda \in \mathbb{C}: q(T) \leq|\lambda| \leq r(T)\} .
$$


(ii) If $r^{-}(T)<r_{1}^{+}(T)$, then

$$
\sigma_{a p}(T)=\left\{\lambda \in \mathbb{C}: r_{1}^{-}(T) \leq|\lambda| \leq r^{-}(T)\right\} \cup\left\{\lambda \in \mathbb{C}: r_{1}^{+}(T) \leq|\lambda| \leq r^{+}(T)\right\} .
$$

Otherwise,

$$
\sigma(T)=\sigma_{a p}(T)=\left\{\lambda \in \mathbb{C}: r_{1}(T) \leq|\lambda| \leq r(T)\right\} .
$$

Proof. See [9, Theorem 3] and [10, Theorem 5].

For every nonzero $x \in \mathcal{H}$, we let $x^{(n)}:=\left(\delta_{n, k} x\right)_{k \in \mathbb{Z}}(n \in \mathbb{Z})$, where $\delta_{n, k}$ is the usual Kronecker-delta symbol. Let $S_{x}$ be the restriction of $S$ to $\widehat{\mathcal{H}}(x)$, the closed linear span of $\left\{\left(B_{n} x\right)^{(n)}: n \in \mathbb{Z}\right\}$ in $l^{2}(\mathbb{Z}, \mathcal{H})$. It is an injective bilateral scalar weighted shift on $\widehat{\mathcal{H}}(x)$ with the weight sequence $\left(\left\|B_{n+1} x\right\| /\left\|B_{n} x\right\|\right)_{n \in \mathbb{Z}}$.

The local resolvent set, $\rho_{T}(x)$, of an operator $T \in \mathcal{L}(\mathcal{H})$ at a point $x \in \mathcal{H}$ is the set of all $\lambda \in \mathbb{C}$ for which there exists an analytic function $\phi: O \rightarrow \mathcal{H}$ on some open neighborhood $O$ of $\lambda$ such that

$$
(T-\mu) \phi(\mu)=x(\mu \in O) .
$$

The local spectrum of $T$ at $x$, denoted by $\sigma_{T}(x)$, is equal to the complement of $\rho_{T}(x)$ in $\mathbb{C}$, and is clearly a closed subset of $\sigma(T)$ (see [1] and [7]).

The following lemma is quoted from [4, Lemma 2.1], and will be used in the sequel (see also [3, Proof of Theorem 2.1]).

Lemma 2.2. For every nonzero $x \in \mathcal{H}$, we have $\sigma_{S}(y)=\sigma_{S_{x}}(y)$ for all $y \in \widehat{\mathcal{H}}(x)$.

Theorem 2.3. If $\left(e_{i}\right)_{i \in \mathrm{I}}$ is an orthonormal basis of $\mathcal{H}$, then

$$
\sigma(S)=\sigma_{a p}(S) \cup\left\{\bigcup_{i \in \mathrm{I}}\left\{\lambda \in \mathbb{C}: q\left(S_{e_{i}}\right) \leq|\lambda| \leq r\left(S_{e_{i}}\right)\right\}\right\} .
$$

Proof. Since each $S_{e_{i}}$ is a bilateral scalar weighted shift, the identity

$$
\sigma\left(S_{e_{i}}\right)=\left\{\lambda \in \mathbb{C}: q\left(S_{e_{i}}\right) \leq|\lambda| \leq r\left(S_{e_{i}}\right)\right\}(i \in \mathrm{I})
$$

follows from Theorem 2.1(i). On the other hand, it follows from Lemma 2.2 and [1, Theorem 2.43] that

$$
\sigma_{s u}\left(S_{e_{i}}\right)=\bigcup_{x \in \widehat{\mathcal{H}}\left(e_{i}\right)} \sigma_{S_{e_{i}}}(x)=\bigcup_{x \in \widehat{\mathcal{H}}\left(e_{i}\right)} \sigma_{S}(x) \subset \sigma_{s u}(S)
$$

for all $i \in \mathrm{I}$. As $\sigma_{p}\left(S_{e_{i}}\right) \subset \sigma_{p}(S)$ for all $i \in \mathrm{I}$, we see that

$$
\sigma\left(S_{e_{i}}\right)=\sigma_{p}\left(S_{e_{i}}\right) \cup \sigma_{s u}\left(S_{e_{i}}\right) \subset \sigma_{p}(S) \cup \sigma_{s u}(S)=\sigma(S)
$$

for all $i \in \mathrm{I}$. Hence,

$$
\bigcup_{i \in \mathrm{I}}\left\{\lambda \in \mathbb{C}: q\left(S_{e_{i}}\right) \leq|\lambda| \leq r\left(S_{e_{i}}\right)\right\} \subset \sigma(S) .
$$

Now, assume that there is $\lambda_{0} \in \sigma(S) \backslash\left\{\bigcup_{i \in \mathrm{I}}\left\{\lambda \in \mathbb{C}: q\left(S_{e_{i}}\right) \leq|\lambda| \leq r\left(S_{e_{i}}\right)\right\}\right\}$. We have

$$
\left(S-\lambda_{0}\right) \widehat{\mathcal{H}}\left(e_{i}\right)=\left(S_{e_{i}}-\lambda_{0}\right) \widehat{\mathcal{H}}\left(e_{i}\right)=\widehat{\mathcal{H}}\left(e_{i}\right)
$$

for all $i \in \mathrm{I}$. Thus, all finite combinations of elements of $\widehat{\mathcal{H}}\left(e_{i}\right)$ 's are in the range of $\left(S-\lambda_{0}\right)$, which is now clearly dense in $l^{2}(\mathbb{Z}, \mathcal{H})$. Therefore, $\lambda_{0} \in \sigma_{a p}(S)$ and the proof is complete. 
In the rest of this section, we assume that $\mathcal{H}=\mathbb{C}^{k}$ and let $\left(e_{i}\right)_{1 \leq i \leq k}$ be the canonical orthonormal basis of $\mathbb{C}^{k}$. In [6, Theorem 2.1], Li, Ji and Sun proved that $S$ is unitarily equivalent to a bilateral operator-weighted shift for which all the weights are $k \times k$ upper-triangular matrices. Thus, we may and shall assume that each $A_{n}$ is a $k \times k$ upper-triangular matrix:

$$
A_{n}=\left[\begin{array}{cccc}
v_{11}^{(n)} & v_{12}^{(n)} & \ldots & v_{1 k}^{(n)} \\
0 & v_{22}^{(n)} & \ldots & v_{2 k}^{(n)} \\
\vdots & \ddots & \ddots & \vdots \\
0 & \ldots & 0 & v_{k k}^{(n)}
\end{array}\right] \quad(n \in \mathbb{Z})
$$

The following gives the complete description of the spectrum of $S$.

Theorem 2.4. The spectrum of $S$ is given by

$$
\sigma(S)=\bigcup_{i=1}^{k}\left\{\lambda \in \mathbb{C}: \min \left(r_{1}\left(T_{i}\right), q\left(S_{e_{i}}\right)\right) \leq|\lambda| \leq \max \left(r\left(T_{i}\right), r\left(S_{e_{i}}\right)\right)\right\},
$$

where each $T_{i}$ is the bilateral scalar weighted shift on $l^{2}(\mathbb{Z})$ with the weight sequence $\left(v_{i i}^{(n)}\right)_{n \in \mathbb{Z}}$.

Proof. It follows from [6, Theorem 2.2] that $\sigma_{a p}(S)=\bigcup_{i=1}^{k} \sigma_{a p}\left(T_{i}\right)$, and so, by Theorem 2.3, we have

$$
\sigma(S)=\bigcup_{i=1}^{k}\left\{\sigma_{a p}\left(T_{i}\right) \cup \sigma\left(S_{e_{i}}\right)\right\}
$$

To finish the proof, it suffices to show that

$$
\sigma_{a p}\left(T_{i}\right) \cup \sigma\left(S_{e_{i}}\right)=\left\{\lambda \in \mathbb{C}: \min \left(r_{1}\left(T_{i}\right), q\left(S_{e_{i}}\right)\right) \leq|\lambda| \leq \max \left(r\left(T_{i}\right), r\left(S_{e_{i}}\right)\right)\right\}
$$

for all $i$.

It is clear that each $B_{n}$ is also a $k \times k$ upper-triangular matrix that has, of course, the following form:

$$
B_{n}=\left[\begin{array}{cccc}
\omega_{11}^{(n)} & \omega_{12}^{(n)} & \ldots & \omega_{1 k}^{(n)} \\
0 & \omega_{22}^{(n)} & \ldots & \omega_{2 k}^{(n)} \\
\vdots & \ddots & \ddots & \vdots \\
0 & \ldots & 0 & \omega_{k k}^{(n)}
\end{array}\right]
$$

Note that, since each $A_{n}$ equals $B_{n+1} B_{n}{ }^{-1}$, we have $v_{i i}^{(n)}=\frac{\omega_{i i}^{(n+1)}}{\omega_{i i}^{(n)}}$ for all $n \in \mathbb{Z}$ and all $i$. On the other hand, each $S_{e_{i}}$ is a bilateral scalar weighted shift with the weight sequence $\left(\sqrt{\frac{\sum_{j=1}^{i}\left|\omega_{j i}^{(n+1)}\right|^{2}}{\sum_{j=1}^{i}\left|\omega_{j i}^{(n)}\right|^{2}}}\right)_{n \in \mathbb{Z}}$. For every $i$, we have

$$
r_{3}^{+}\left(T_{i}\right) \leq r_{3}^{+}\left(S_{e_{i}}\right) .
$$


Indeed, we have

$$
\begin{aligned}
r_{3}^{+}\left(S_{e_{i}}\right) & =\limsup _{n \rightarrow+\infty}\left\{\frac{\sum_{j=1}^{i}\left|\omega_{j i}^{(n)}\right|^{2}}{\sum_{j=1}^{i}\left|\omega_{j i}^{(0)}\right|^{2}}\right\}^{\frac{1}{2 n}} \\
& \geq \limsup _{n \rightarrow+\infty}\left|\omega_{i i}^{(n)}\right|^{\frac{1}{n}}=\limsup _{n \rightarrow+\infty}\left\{v_{i i}^{(0)} v_{i i}^{(1)} \ldots v_{i i}^{(n-1)}\right\}^{\frac{1}{n}} \\
& =r_{3}^{+}\left(T_{i}\right) .
\end{aligned}
$$

Similarly, we have

$$
r_{2}^{-}\left(S_{e_{i}}\right) \leq r_{2}^{-}\left(T_{i}\right)
$$

for all $i$.

Now, we fix an integer $i$ such that $(1 \leq i \leq k)$ and, in view of Theorem 2.1(ii), shall consider two cases.

Case 1. Assume that $r^{-}\left(T_{i}\right)<r_{1}^{+}\left(T_{i}\right)$ and recall that, in this case, we have

$$
\sigma_{a p}\left(T_{i}\right)=\left\{\lambda \in \mathbb{C}: r_{1}^{-}\left(T_{i}\right) \leq|\lambda| \leq r^{-}\left(T_{i}\right)\right\} \cup\left\{\lambda \in \mathbb{C}: r_{1}^{+}\left(T_{i}\right) \leq|\lambda| \leq r^{+}\left(T_{i}\right)\right\}
$$

(see Theorem 2.1(ii)). Moreover, in view of the above inequalities, we have

$$
q\left(S_{e_{i}}\right) \leq r_{2}^{-}\left(S_{e_{i}}\right) \leq r_{2}^{-}\left(T_{i}\right) \leq r^{-}\left(T_{i}\right)<r_{1}^{+}\left(T_{i}\right) \leq r_{3}^{+}\left(T_{i}\right) \leq r_{3}^{+}\left(S_{e_{i}}\right) \leq r\left(S_{e_{i}}\right) .
$$

From these inequalities and (2.3), it follows that

$$
\begin{aligned}
& \left\{\lambda \in \mathbb{C}:|\lambda|=r_{2}^{-}\left(T_{i}\right)\right\} \subset\left\{\lambda \in \mathbb{C}: r_{1}^{-}\left(T_{i}\right) \leq|\lambda| \leq r^{-}\left(T_{i}\right)\right\} \cap \sigma\left(S_{e_{i}}\right), \\
& \left\{\lambda \in \mathbb{C}:|\lambda|=r_{3}^{+}\left(T_{i}\right)\right\} \subset\left\{\lambda \in \mathbb{C}: r_{1}^{+}\left(T_{i}\right) \leq|\lambda| \leq r^{+}\left(T_{i}\right)\right\} \cap \sigma\left(S_{e_{i}}\right) .
\end{aligned}
$$

From these inclusions, (2.1) and (2.2), it is clearly seen that the desired identity, (2.4), holds.

Case 2. Now, assume that $r^{-}\left(T_{i}\right) \geq r_{1}^{+}\left(T_{i}\right)$. By Theorem 2.1(ii), we have

$$
\sigma_{a p}\left(T_{i}\right)=\left\{\lambda \in \mathbb{C}: r_{1}\left(T_{i}\right) \leq|\lambda| \leq r\left(T_{i}\right)\right\} .
$$

In view of (2.5) and (2.6), we clearly have

$$
q\left(S_{e_{i}}\right) \leq r\left(T_{i}\right) \text { and } r_{1}\left(T_{i}\right) \leq r\left(S_{e_{i}}\right) .
$$

From these, we see that $\sigma_{a p}\left(T_{i}\right)$ intersects $\sigma\left(S_{e_{i}}\right)$ and the desired identity, (2.4), holds in this case too.

\section{Additional facts And COMments}

Recall that an operator $T \in \mathcal{L}(\mathcal{H})$ is said to be hyponormal if $T^{*} T \geq T T^{*}$ and note that the bilateral operator-weighted shift $S$ is hyponormal if and only if $A_{n+1}^{*} A_{n+1} \geq A_{n} A_{n}^{*}$ for all $n \in \mathbb{Z}$.

An operator $T \in \mathcal{L}(\mathcal{H})$ is said to satisfy Dunford's condition $(C)$ provided that, for every closed subset $F$ of $\mathbb{C}$, the linear subspace

$$
\mathcal{H}_{T}(F):=\left\{x \in \mathcal{H}: \sigma_{T}(x) \subset F\right\}
$$

is closed. It is well known that all hyponormal operators enjoy Dunford's condition (C) (see [8, Proposition 1]). 
The following is a slight clarification of [4, Theorem 4.2].

Theorem 3.1. If $\left(e_{i}\right)_{i \in \mathrm{I}}$ is an orthonormal basis of $\mathcal{H}$ and $S$ satisfies Dunford's condition $(C)$, then

$$
\sigma(S)=\operatorname{cl}\left\{\bigcup_{i \in \mathrm{I}}\left\{\lambda \in \mathbb{C}: r_{2}^{-}\left(S_{e_{i}}\right) \leq|\lambda| \leq r_{3}^{+}\left(S_{e_{i}}\right)\right\}\right\} .
$$

Proof. We shall proceed as in [4 in order to prove this result. It follows from Lemma 2.2 that each $S_{e_{i}}$ satisfies Dunford's condition $(C)$ (see also [1, Theorem 2.79]). Hence,

$$
\sigma\left(S_{e_{i}}\right)=\sigma_{S_{e_{i}}}\left(e_{i}{ }^{(0)}\right)=\left\{\lambda \in \mathbb{C}: r_{2}^{-}\left(S_{e_{i}}\right) \leq|\lambda| \leq r_{3}^{+}\left(S_{e_{i}}\right)\right\}
$$

for all $i \in \mathrm{I}$, and

$$
F:=\operatorname{cl}\left\{\bigcup_{i \in \mathrm{I}}\left\{\lambda \in \mathbb{C}: r_{2}^{-}\left(S_{e_{i}}\right) \leq|\lambda| \leq r_{3}^{+}\left(S_{e_{i}}\right)\right\}\right\} \subset \sigma(S) .
$$

Again use Lemma 2.2 to see that

$$
\widehat{\mathcal{H}}\left(e_{i}\right)=\left\{\widehat{\mathcal{H}}\left(e_{i}\right)\right\}_{S_{e_{i}}}\left(\sigma\left(S_{e_{i}}\right)\right) \subset\left\{l^{2}(\mathbb{Z}, \mathcal{H})\right\}_{S}(F)
$$

for all $i \in \mathrm{I}$. As the closed linear span of $\widehat{\mathcal{H}}\left(e_{i}\right)$ 's coincides with $l^{2}(\mathbb{Z}, \mathcal{H})$, we see that $\left\{l^{2}(\mathbb{Z}, \mathcal{H})\right\}_{S}(F)=l^{2}(\mathbb{Z}, \mathcal{H})$ and $\sigma(S)=\sigma\left(S_{\mid\left\{l^{2}(\mathbb{Z}, \mathcal{H})\right\}_{S}(F)}\right) \subset F$ (see 1, Theorem 2.71]). This concludes the proof.

As consequences of this theorem, we derive in particular the following corollaries, which improve some results that have appeared in [5].

Corollary 3.2. If $\left(e_{i}\right)_{i \in \mathrm{I}}$ is an orthonormal basis of $\mathcal{H}$ and $S$ is hyponormal, then

$$
\sigma(S)=\operatorname{cl}\left\{\bigcup_{i \in \mathrm{I}}\left\{\lambda \in \mathbb{C}: m\left(S_{e_{i}}\right) \leq|\lambda| \leq\left\|S_{e_{i}}\right\|\right\}\right\} .
$$

Proof. Note that, since $S_{e_{i}}$ coincides with $S$ when restricted to $\widehat{\mathcal{H}}\left(e_{i}\right)(i \in \mathrm{I}), S_{e_{i}}$ is also hyponormal. By [10], we have

$$
\begin{gathered}
r_{1}^{-}\left(S_{e_{i}}\right)=r_{2}^{-}\left(S_{e_{i}}\right)=r_{3}^{-}\left(S_{e_{i}}\right)=r^{-}\left(S_{e_{i}}\right)=m\left(S_{e_{i}}\right), \\
r_{1}^{+}\left(S_{e_{i}}\right)=r_{2}^{+}\left(S_{e_{i}}\right)=r_{3}^{+}\left(S_{e_{i}}\right)=r^{+}\left(S_{e_{i}}\right)=\left\|S_{e_{i}}\right\|
\end{gathered}
$$

for all $i \in \mathrm{I}$. Now, the desired result follows from Theorem 3.1 ,

The next result is an immediate consequence of Theorem 3.1. It is a generalization of Herrero's result to the setting of bilateral operator-weighted shifts of multiplicity $k$ which satisfy Dunford's condition $(C)$. It gives precise information about the location of the $k$ annuli appearing in Herrero's result.

Corollary 3.3. Assume that $\mathcal{H}=\mathbb{C}^{k}$ and let $\left(e_{i}\right)_{1 \leq i \leq k}$ be the canonical orthonormal basis of $\mathbb{C}^{k}$. If $S$ satisfies Dunford's condition $(C)$, then

$$
\sigma(S)=\bigcup_{i=1}^{k}\left\{\lambda \in \mathbb{C}: r_{2}^{-}\left(S_{e_{i}}\right) \leq|\lambda| \leq r_{3}^{+}\left(S_{e_{i}}\right)\right\} .
$$

\section{ACKNOWLEDGMENTS}

The author thanks Professor T. J. Ransford for useful comments and fruitful discussions. 


\section{REFERENCES}

[1] P. Aiena, Fredholm and local spectral theory, with applications to multipliers, Kluwer, Dordrecht, 2004. MR2070395 (2005e:47001)

[2] A. Ben-Artzi and I. Gohberg, Dichotomy, discrete Bohl exponents, and spectrum of block weighted shifts, Integral equations and Operator Theory, 14 (1991) 613-677. MR1118967 (93e:47033)

[3] A. Bourhim and C. E. Chidume, The single-valued extension property for bilateral operator weighted shifts, Proc. Amer. Math. Soc. 133 (2005), 485-491. MR2093072

[4] A. Bourhim, Local spectra of bilateral operator weighted shifts, Rend. Circ. Mat. Palermo (2) Suppl. No. 73 (2004), 195-206. MR2078176 (2005d:47054)

[5] D. Herrero, Spectral pictures of hyponormal bilateral operator weighted shifts, Proc. Amer. Math. Soc. 109 (1990) 753-763. MR:1014644 (90k:47050)

[6] J. X. Li, Y. Q. Ji and S. L. Sun, The essential spectrum and Banach reducibility of operator weighted shifts, Acta Math. Sinica, English Series, Vol. 17, 3 (2001) 413-424. MR1852955 (2002g:47063)

[7] K. B. Laursen and M. M. Neumann, An introduction to local spectral theory, London Mathematical Society Monograph New Series 20 (2000). MR1747914 (2001k:47002)

[8] M. Radjabalipour, Ranges of hyponormal operators, Illinois J. Math. 21 (1977) 70-75. MR0448140 (56:6449)

[9] W. C. Ridge, Approximate point spectrum of a weighted shift, Trans. Amer. Math. Soc. 147 (1970) 349-356. MR0254635 (40:7843)

[10] A. L. Shields, Weighted shift operators and analytic function theory, in Topics in Operator Theory, Mathematical Surveys, No. 13 (ed. C. Pearcy), pp. 49-128. American Mathematical Society, Providence, Rhode Island 1974. MR0361899 (50:14341)

Département de Mathématiques et de Statistique, Université Laval, Québec, Québec, CANADA G1K 7P4

E-mail address: bourhim@mat.ulaval.ca 